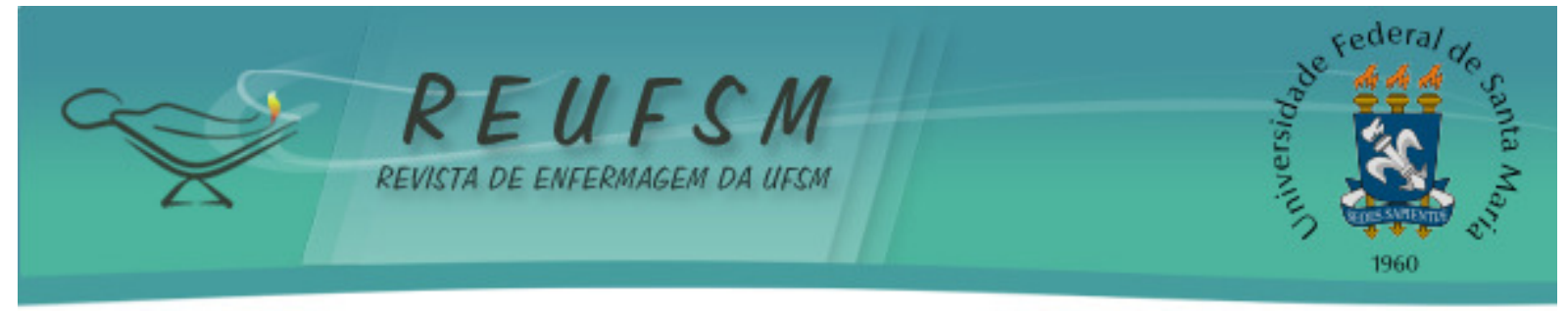

NOTA PRÉVIA

\title{
HUMANIZAÇÃO DA ATENÇÃO PRÉ-NATAL NA PRÁXIS DOS PROFISSIONAIS DE SAÚDE: NOTA PRÉVIA
}

\section{HUMANIZATION OF PRENATAL CARE IN THE PRAXIS OF HEALTH PROFESSIONALS: INTRODUCTORY NOTE}

\section{HUMANIZACIÓN DE LA ATENCIÓN PRENATAL EN LA PRAXIS DE PROFISIONALES DE SALUD: NOTA INTRODUCTORIA}

\author{
Camila Nunes Barreto ${ }^{1}$ \\ Luiza Cremonese ${ }^{2}$ \\ Lisie Alende Prates ${ }^{3}$ \\ Juliane Scarton ${ }^{4}$ \\ Lúcia Beatriz Ressel ${ }^{5}$
}

Doi: $10.5902 / 2179769212970$

RESUMO: Objetivo: conhecer como ocorre a aproximação dos pressupostos de humanização das políticas públicas e programas de saúde, propostos pelo Ministério da Saúde, na práxis da atenção pré-natal de risco habitual. Método: estudo de campo, descritivo, com abordagem qualitativa e vertente antropológica. 0 cenário do estudo será composto por Estratégias de Saúde da Família. Os sujeitos serão enfermeiros e médicos que desenvolvam ações na abrangência da atenção pré-natal. Os dados serão coletados por meio de observação participante e entrevista semiestruturada e, após, serão analisados por meio da proposta operativa. Resultados esperados: espera-se que este estudo promova discussões e reflexões que auxiliem na compreensão do contexto da humanização na atenção pré-natal e possa contribuir na qualificação da assistência prestada à gestante. Descritores: Cuidado pré-natal; Humanização da assistência; Enfermagem.

ABSTRACT: Aim: to identify the process of approximation of the presuppositions of humanization and the public policies and health programs, proposed by the Ministry of Health, in the praxis of low-risk prenatal care. Method: descriptive field study, using a qualitative approach and anthropological perspective. The setting of the study will consist of Family Health Strategies. The subjects will be nurses and doctors who develop actions in the scope of prenatal care. Data will be collected through participant observation and semi-structured interview and, afterwards, analyzed using the operative proposal. Expected Results: this study is expected to promote discussions and reflections that promote the understanding of the context of humanization in prenatal care and can contribute to the qualification of the care to pregnant women.

Descriptors: Prenatal care; Humanization of assistance; Nursing.

RESUMEN: Objetivo: conocer cómo ocurre la aproximación de los presupuestos de la humanización de las políticas públicas y programas de salud propuestos por el Ministerio

\footnotetext{
${ }^{1}$ Enfermeira, Graduação em Enfermagem pela Universidade Federal de Santa Maria, Santa Maria, Rio Grande do Sul, Brasil, e-mail: camilabarreto_6@msn.com

${ }^{2}$ Enfermeira, Graduação em Enfermagem pela Universidade Federal de Santa Maria, Santa Maria, Rio Grande do Sul, Brasil, e-mail: lu_cremonese@hotmail.com

${ }^{3}$ Enfermeira, Graduação em Enfermagem pela Universidade Federal do Pampa, Santa Maria, Rio Grande do Sul, Brasil, e-mail: lisiealende@hotmail.com

${ }^{4}$ Enfermeira, Graduação em Enfermagem pela Universidade Regional do Noroeste do Estado do Rio Grande do Sul, Santa Maria, Rio Grande do Sul, Brasil, e-mail: juliscarton10@hotmail.com

${ }^{5}$ Enfermeira, Doutorado em Enfermagem pela Universidade de São Paulo, Santa Maria, Rio Grande do Sul, Brasil, e-mail: lbressel208@yahoo.com.br
} 


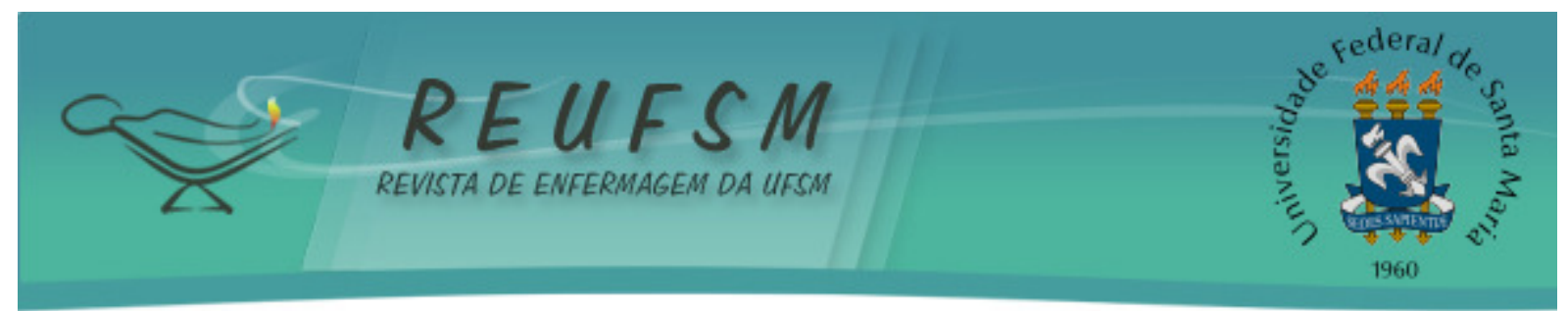

de Salud, en la práctica de la atención prenatal de riesgo habitual. Método: estudio de campo, descriptivo, con enfoque cualitativo y antropológico. El ámbito del estudio está compuesto por la Estrategia de Salud de la Familia. Los sujetos son enfermeras y médicos que desarrollan acciones en el ámbito de la atención prenatal. Los datos se recogen mediante la observación participante y la entrevista semi-estructurada, y después se analizarán mediante la propuesta operativa. Resultados esperados: se espera que este estudio promueva discusiones y reflexiones que ayuden a comprender el contexto de la humanización en la atención prenatal y pueda contribuir a la cualificación de la atención a las mujeres embarazadas.

Descriptores: Atención prenatal; Humanización de la atención; Enfermería.

\section{INTRODUÇÃO}

A humanização da atenção pré-natal é o primeiro passo na busca de um atendimento integral à gestante. O Ministério da Saúde (MS), com o intuito de qualificar as ações ofertadas, propõe por meio das políticas públicas e programas de saúde, transformações e renovações na assistência à saúde da mulher. Destaca-se a Política Nacional de Humanização, a qual busca a transversalidade na atenção e na gestão do Sistema Único de Saúde. ${ }^{1}$

Nesta direção, propôs-se para esta pesquisa o seguinte objetivo: conhecer como ocorre a aproximação dos pressupostos de humanização das políticas públicas e programas de saúde propostos pelo MS na práxis da atenção pré-natal de risco habitual.

\section{MÉTODO}

A trajetória metodológica prevê o estudo de campo, de caráter descritivo exploratório, com abordagem qualitativa e vertente antropológica. O campo para realização do estudo será composto por Estratégias de Saúde da Família (ESF) da rede básica de saúde de um município do interior do Rio Grande do Sul. Os sujeitos do estudo serão enfermeiros e médicos. Os critérios de inclusão compreendem: enfermeiros e médicos que desenvolvam ações referentes à atenção pré-natal nas Estratégias de Saúde da Família. Os critérios de exclusão serão: enfermeiros e médicos que estejam afastados do serviço no momento da pesquisa.

A produção dos dados ocorrerá por meio das técnicas de observação participante e entrevista semiestruturada. A análise dos dados será fundamentada na proposta operativa. $^{2} \mathrm{O}$ projeto foi aprovado pelo Comitê de Ética em Pesquisa com Seres Humanos sob número do parecer 513.040, conforme preconizado pela Resolução 466/12.

Espera-se que este estudo promova discussões e reflexões que auxiliem na compreensão do contexto da humanização na atenção pré-natal. Ainda, almeja-se resultados que aproximem a humanização preconizada nas políticas públicas e programas de saúde da práxis dos profissionais de saúde e com isso, oferecendo subsídios para a qualificação da assistência prestada no cuidado pré-natal.

\section{REFERÊNCIAS}

1. Brasil. Ministério da Saúde. Política Nacional de Humanização. Brasília: Ministério da Saúde; 2003.

2. Minayo MCS. O desafio do conhecimento: pesquisa qualitativa em saúde. São Paulo: Hucitec; 2010. 


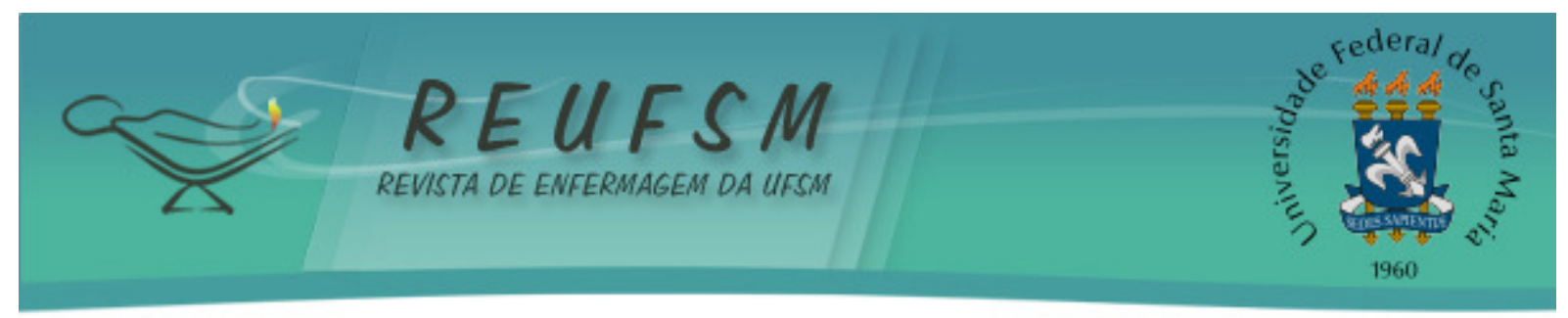

Data de recebimento: 20/02/2014

Data de aceite: $13 / 11 / 2014$

Contato com autor responsável: Camila Nunes Barreto

Endereço postal: Programa de Pós Graduação em Enfermagem - Centro de Ciências da Saúde - Universidade Federal de Santa Maria - Avenida Roraima n 1000, Prédio 26 - Cidade Universitária, CEP: 97105-900, Santa Maria (RS), Brasil.

E-mail: camilabarreto_6@msn.com 